\title{
FHL3 links cell growth and self-renewal by modulating SOX4 in glioma
}

\author{
Wei Han ${ }^{1}$ Peishan $\mathrm{Hu}^{1} \cdot$ Fan $\mathrm{Wu}^{1,3} \cdot$ Shanshan Wang ${ }^{1} \cdot$ Yan $\mathrm{Hu}^{1} \cdot$ Shanshan $\mathrm{Li}^{1} \cdot$ Tao Jiang $^{3} \cdot$ Boqin Qiang ${ }^{1}$. \\ Xiaozhong Peng ${ }^{1,2}$
}

Received: 30 March 2018 / Revised: 13 May 2018 / Accepted: 5 June 2018 / Published online: 28 June 2018

๑) ADMC Associazione Differenziamento e Morte Cellulare 2018

\begin{abstract}
Differentiation status significantly affects the properties of malignant glioma cells, with non-stem cells inducing tumor enlargement and stem-like cells driving tumor initiation and treatment resistance. It is not completely understood how the same protein can have a distinct role in these cell populations. Here, we report that four and a half LIM domain protein 3 (FHL3) has an inhibitory effect on proliferation in non-stem glioma cells and a non-proliferative effect in glioma stem cells (GSCs). In GSCs, we show that FHL3 interacts with the Smad2/3 protein complex at the SOX4 promoter region, inhibits SOX4 transcriptional activity by recruiting PPM1A phosphatase to Smad2/3, and then suppresses GSC tumor sphere formation and self-renewal in vitro and in vivo via downregulation of SOX2 expression. Altogether, these findings highlight the role of FHL3 as a stemness-suppressor in regulation of the Smad2/3-SOX4-SOX2 axis in glioma.
\end{abstract}

\section{Introduction}

Gliomas account for $27 \%$ of all brain tumors and $80 \%$ of all malignant brain tumors. WHO grade III anaplastic

These authors contributed equally: Wei Han, Peishan Hu.

Edited by P. Salomoni

Electronic supplementary material The online version of this article (https://doi.org/10.1038/s41418-018-0152-1) contains supplementary material, which is available to authorized users.

Boqin Qiang

chiangbq@imicams.ac.cn

$\triangle$ Xiaozhong Peng pengxiaozhong@pumc.edu.cn peng_xiaozhong@163.com

1 State Key Laboratory of Medical Molecular Biology, Department of Molecular Biology and Biochemistry, Institute of Basic Medical Sciences, Medical Primate Research Center, Neuroscience Center, Chinese Academy of Medical Sciences, School of Basic Medicine Peking Union Medical College, 100005 Beijing, China

2 Institute of Medical Biology, Chinese Academy of Medical Sciences, Chinese Academy of Medical Science and Peking Union Medical College, 650118 Kunming, China

3 Department of Neurosurgery, Beijing Tiantan Hospital, 100050 Beijing, China astrocytomas (AAs) and grade IV glioblastoma multiforme (GBMs) are highly invasive tumors and make up approximately three-quarters of all gliomas [1]. Cell populations of different proliferative, stem-like and tumorigenic states frequently coexist in GBM. Enrichment of glioma stem cells (GSCs) may occur as a result of either self-renewal via symmetric divisions or the conversion of non-stem glioma cells to stem-like cells. GSCs were among the first cancer stem cells to be described for solid tumors and the existence of GSCs is now widely accepted [2]. The clinical cancer relevance of GSCs relies on the observation that the GSC population is more resistant to frequently used anti-glioma therapies than the non-stem glioma cell population [3].

GSCs are functionally distinct from non-stem glioma cells and share several common characteristics with stem cells including the ability to self-renew as well as an increased capacity to form glioma spheres, express stem cell markers (such as CD133, NESTIN, and SOX2), differentiate into multiple lineages (i.e., neurons or glial cells), and regenerate the original tumor in vivo. The functional diversity that exists between GSCs and non-stem glioma cells can derive from genetic, epigenetic, and metabolic environmental differences among tumor cells [4-6]. Therefore, an understanding of the specific functions of non-stem and stem-like glioma cells as well as the mechanisms regulating oncogenes and tumor suppressor genes in these two cell types will help to develop a more 
A

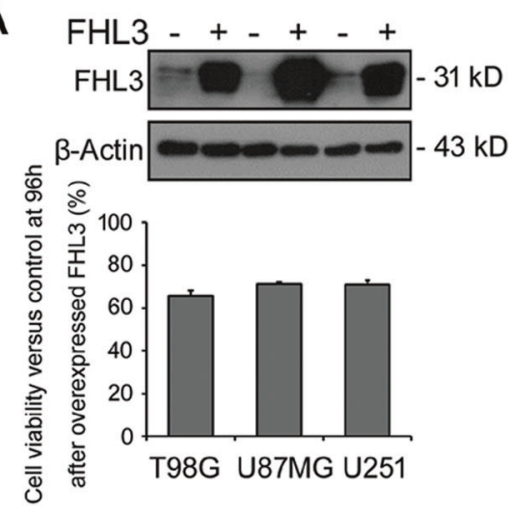

B

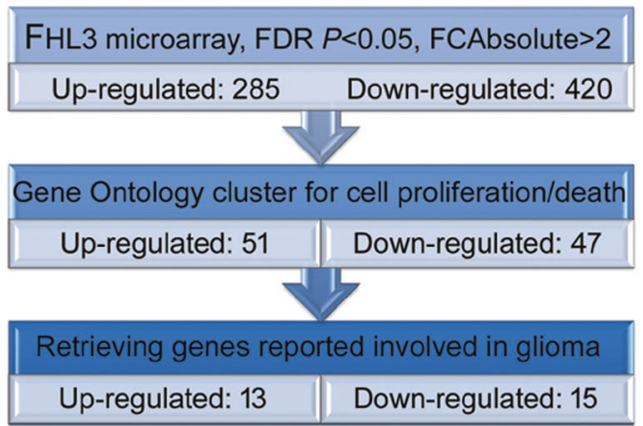

C

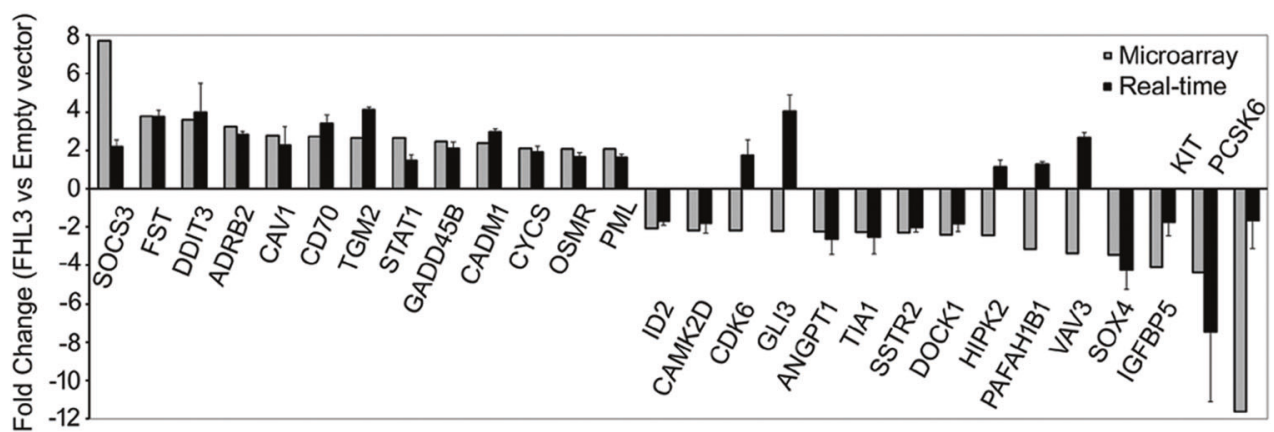

D

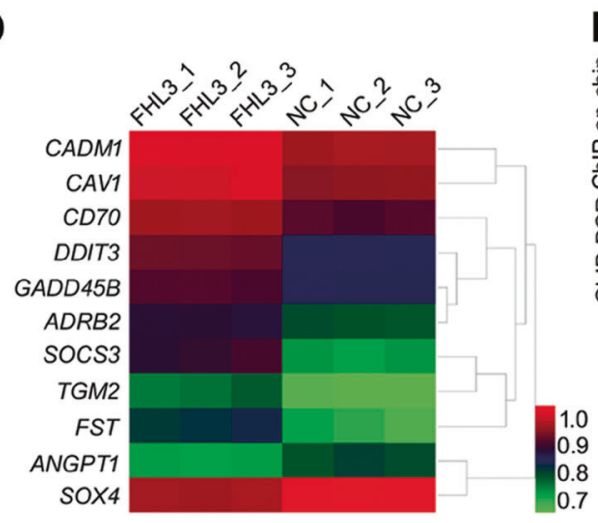

Fig. 1 FHL3 regulates the target genes SOX4, CAV1, and DDIT3 in glioma cells. a Glioma cell lines (T98G, U87MG, and U251) were transfected with PLVX empty vector $(-)$ or FHL3 overexpression plasmid (+). Lysates were collected $48 \mathrm{~h}$ post-transfection and immunoblotted for the indicated proteins. $\beta$-Actin was used as a loading control. The bar graph shows cell viability relative to the control groups $96 \mathrm{~h}$ post-transfection. b Schematic illustration of the procedure used to screen and refine the set of FHL3-regulated target genes identified by three independent glioma microarray data replicates. c Twenty-eight indicated genes reported to be involved in glioma were assessed by microarray (gray bars) and real-time PCR (black bars). GAPDH was used as a housekeeping gene. d Heatmaps illustrating the expression

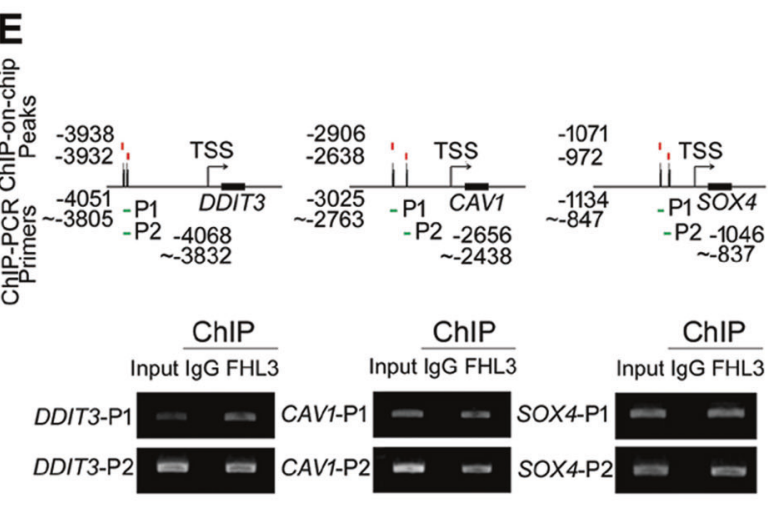

profiles of the 11 differentially expressed genes verified by microarray experiments ( $n=3$ for each biological replicate). e Soluble chromatin was subjected to immunoprecipitation with IgG or anti-FHL3 antibodies in T98G cells. We designed two pairs of primers (P1 and P2) to amplify the predicted binding regions upstream of each gene, as peaks were identified in these regions in the ChIP-on-chip assay. The locations of the peaks identified by the ChIP-on-chip assay are denoted as short red bars. Immunoprecipitated DNA was PCR amplified with primers (locations indicated with short green bars) that annealed to the proximal region of the DDIT3, CAV1, or SOX4 promoters. The lengths of the amplified fragments are $247 \mathrm{bp}$ (DDIT3-P1), $237 \mathrm{bp}$ (DDIT3-P2), $263 \mathrm{bp}$ (CAV1P1), 219 bp (CAV1-P2), 288 bp (SOX4-P1), and 210 bp (SOX4-P2)

a novel target gene of PCBP2 and is downregulated in glioma tissues [7]. FHL3 mainly displayed proapoptotic activity and inhibited glioma growth in vivo, however the detailed mechanism driving FHL3 downstream regulation in gliomas and its role in GSCs remained unknown. first time that four-and-a-half LIM-only protein 3 (FHL3) is 
A

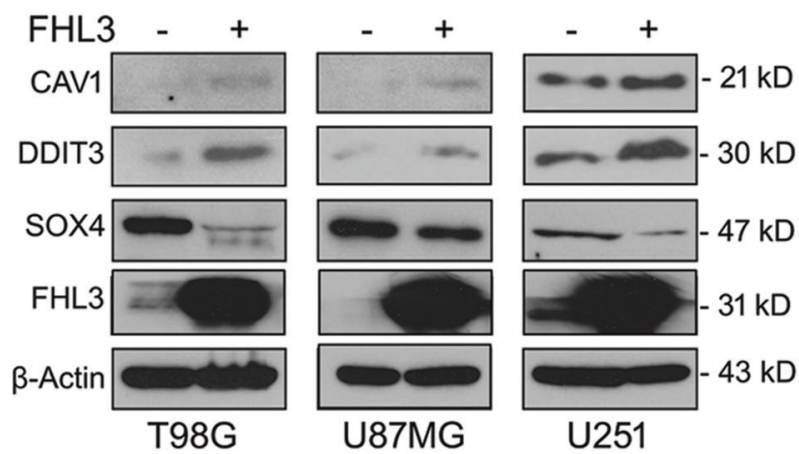

C

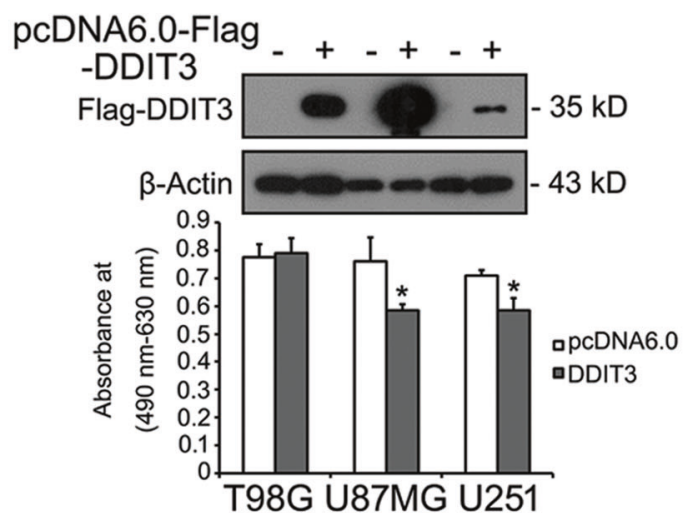

E
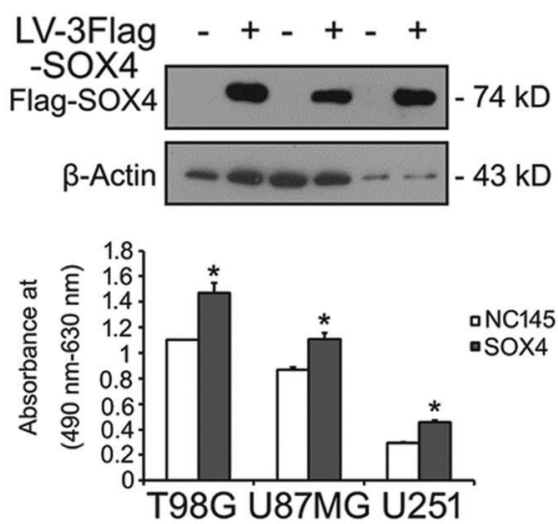

Fig. 2 FHL3 inhibits glioma cell proliferation mainly through the downregulation of SOX4 expression. a Representative western blot showing CAV1, DDIT3, SOX4, and FHL3 protein levels in FHL3overexpressing $(+)$ glioma cell lines. b, c Western blot analysis of T98G, U87MG, and U251 glioma cell lines transfected with pcDNA6.0-Flag-CAV1 (b), pcDNA6.0-Flag-DDIT3 (c) or control vector (-). An anti-Flag antibody was used to detect target gene overexpression. Bar graphs show the results of MTS assays in the same three glioma cell lines $96 \mathrm{~h}$ after transfection with plasmids. d, e Western blot analysis of SOX4 knockdown and overexpression in T98G, U87MG, and U251 glioma cell lines following lentiviral
B

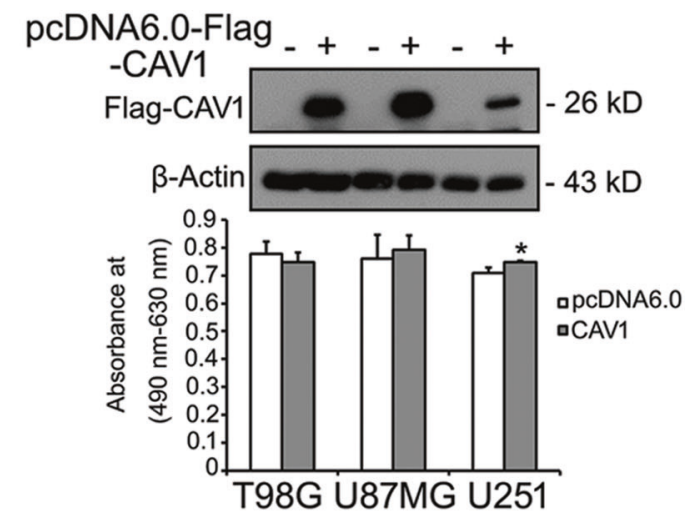

D

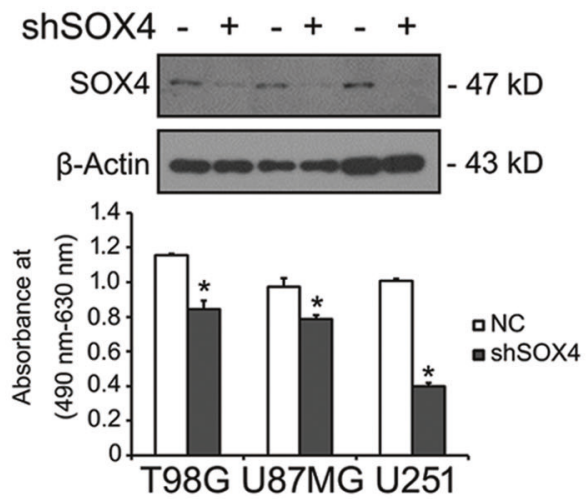

$\mathbf{F}$

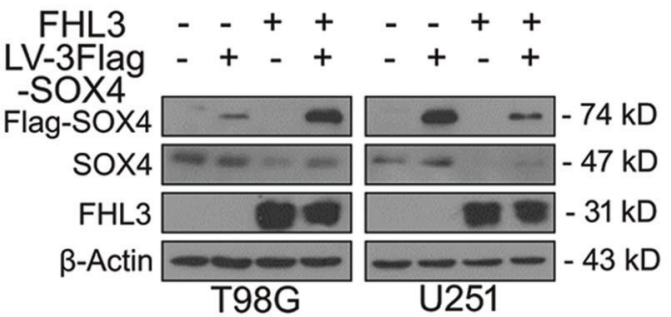

G

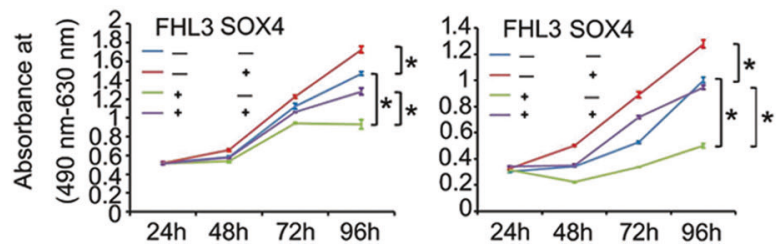

infection with shSOX4 (d), LV-3Flag-SOX4 (e), or a control (-). Anti-SOX4 and anti-Flag antibodies were separately used to detect SOX4 knockdown and overexpression, respectively. Bar graphs show the results of MTS assays performed $96 \mathrm{~h}$ after lentiviral infection in the same three glioma cell lines. f Western blot showing SOX4 and FHL3 protein levels in T98G and U251 glioma cell lines overexpressing either FHL3 or SOX4 alone or co-overexpressing FHL3 and SOX4. g Growth curves in T98G and U251 glioma cells overexpressing either FHL3 or SOX4 alone or co-overexpressing both FHL3 and SOX4. Data are presented as the mean \pm SD of three independent experiments. $* P<0.05$ 
Here, we have assessed the FHL3 target gene SOX4 by gene expression microarray and ChIP-on-chip analysis in non-stem glioma cells and glioma stem cells. We showed that FHL3 overexpression prevented the proliferation of non-stem glioma cells but not glioma stem cells. We found that FHL3 diminished the self-renewal capacity of GSCs and interacted with the transcription factors $\operatorname{Smad} 2 / 3$ and phosphatase PPM1A, thus inhibiting the Smad2/ 3-SOX4-SOX2 axis. In general, our results shed light on some crucial functions of FHL3 in mediating the selfrenewal of glioma stem cells and regulating the growth of non-stem glioma cells through SOX4.

\section{Results}

\section{SOX4 is a novel FHL3 target gene in glioma cells}

We transfected either an FHL3-overexpression construct or an empty vector control into T98G, U87MG, and U251 glioma cell lines (Fig. 1a). In agreement with our previous results [7], an MTS (3-(4,5-dimethylthiazol-2-yl)-5-(3-carboxymethoxyphenyl)-2-(4-sulfophenyl)-2H-tetrazolium, inner salt) assay showed that the viability of glioma cells was reduced to $65-72 \%$ following $96 \mathrm{~h}$ of FHL3 overexpression (Fig. 1a). To investigate FHL3 target genes in glioma cells, we conducted a gene expression microarray analysis. The FHL3 overexpressed T98G glioma cell line was used as the experimental model. We conservatively established a minimum of a twofold difference between the FHL3 and control groups with an FDR (false discovery rate)-adjusted $P$ value of $<0.05$ and identified 285 upregulated and 420 downregulated genes that met the threshold in all microarray analyses from three independent groups (Fig. 1b). The differentially expressed genes were analyzed by gene ontology (Supplementary Figure 1) for association with the 12 biological processes. Among these biological processes, 98 differentially expressed genes, including 51 upregulated and 47 downregulated genes, were enriched for cell proliferation and cell death processes (Fig. 1b). We searched the literature related to the genes enriched in these two biological processes and found that 28 genes were reported to be associated with glioma (Fig. 1b). Then, we selected these 28 genes for confirmation by real-time PCR analyses. Although the results for CDK6, GLI3, HIPK2, $P A F A H 1 B 1$ and VAV3 were unexpectedly contradictory to previous microarray results, most of the results were consistent (Fig. 1c). Eleven genes displayed the same trend and a greater than twofold difference by both real-time PCR and microarray analysis. The nine upregulated genes were SOCS3, CAV1, CD70, FST, CADM1, DDIT3, TGM2, $A D R B 2$, and $G A D D 45 B$, and the two downregulated genes were SOX4 and ANGPT1 (Fig. 1d).
FHL3 comprises four and a half LIM domains, which mediate interactions with some transcription factors such as CREB [8], BKLF/KLF3 [9], MZF-1 [10], and MyoD [11]. These factors can form complexes that increase promoter occupancy in order to regulate the transcription of downstream genes. Therefore, we hypothesized that FHL3 could also regulate the transcription of these 11 genes by binding to their promoter regions via complexes with other transcription factors. Interestingly, the upstream sequences of SOX4, CAV1, and DDIT3 were highly enriched in ChIP-onchip assays (data not shown). ChIP-PCR was used to detect FHL3 occupancy within the regions flanking the SOX4, $C A V 1$, or DDIT3 promoters. Six pairs of primers were designed to amplify the six peaks that were enriched in the ChIP-on-chip assays (Fig. 1e).

\section{FHL3 suppresses glioma cell proliferation by inhibiting SOX4}

We next examined the effect of FHL3 overexpression on SOX4, CAV1, and DDIT3 protein expression in three glioma cell lines. As shown in Fig. 2a, upregulation of FHL3 resulted in the significant downregulation of SOX4 expression and the upregulation of CAV1 and DDIT3 protein expression. Then, we determined which proteins could affect glioma cell proliferation. Compared to CAV1 or DDIT3 overexpression, SOX4 knockdown in glioma cells significantly hindered cell growth within $96 \mathrm{~h}$ (Fig. 2b-d). We also found that SOX4 overexpression could promote cell growth (Fig. 2e). We then asked whether SOX4 is involved in mediating FHL3-induced inhibition of glioma cell proliferation. For these assays, we chose the two cell lines with the highest SOX4 overexpression, T98G and U251. Western blotting revealed that Flag-tagged SOX4 and FHL3 were overexpressed and simultaneously upregulated, respectively, following lentiviral infection (Fig. 2f). MTS assays demonstrated that cell proliferation following co-overexpression of FHL3 and Flag-SOX4 was closer to the proliferation of control cells than FHL3-overexpressing cells (Fig. $2 \mathrm{~g}$ ). These data indicate that the inhibitory effect of FHL3 is dependent on SOX4 downregulation in glioma cells.

\section{FHL3 negatively regulates SOX4 transcriptional activity and TGF- $\beta$-responsive transcription in TGF- $\beta 1$-dependent and TGF- $\beta 1$-independent manners}

How does FHL3 block SOX4 expression? It has previously been reported that FHL1, FHL2, and FHL3 physically and functionally interact with $\mathrm{Smad} 2, \mathrm{Smad} 3$, and Smad4 to increase transforming growth factor- $\beta 1$ (TGF- $\beta 1$ ) responsive transcription [12]. SOX4 is a downstream target of TGF- $\beta 1$ signaling [13]. Thus, we examined whether FHL3 
A

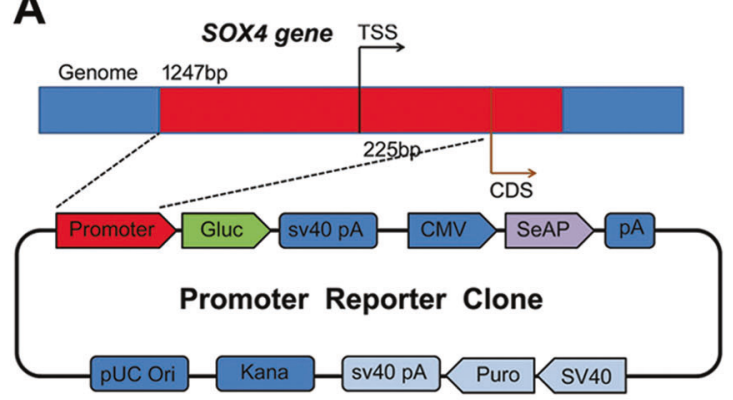

C

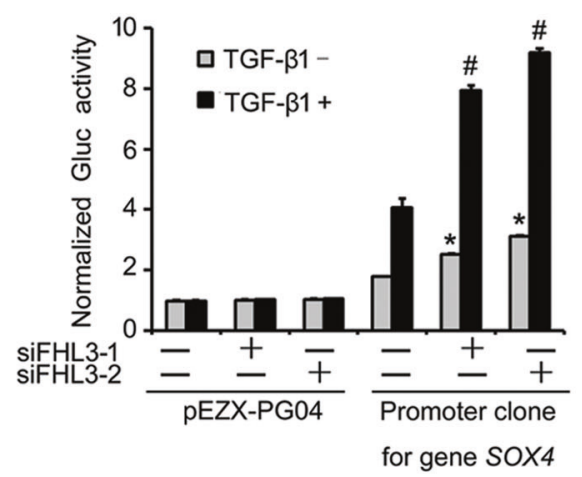

E

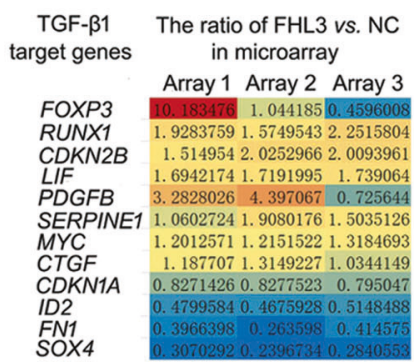

Min

G

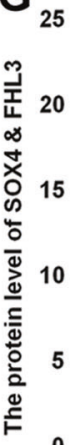

$\operatorname{Max}$
B

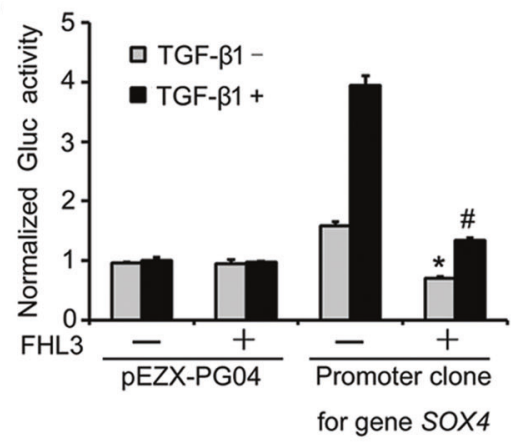

D

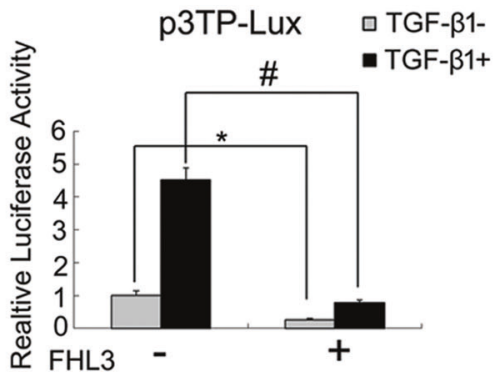

F Spearman-Correlation: -0.3226 $P$-value: $4.751 \mathrm{e}-05$ TCGA GBM Sample Size: ( $N=153)$

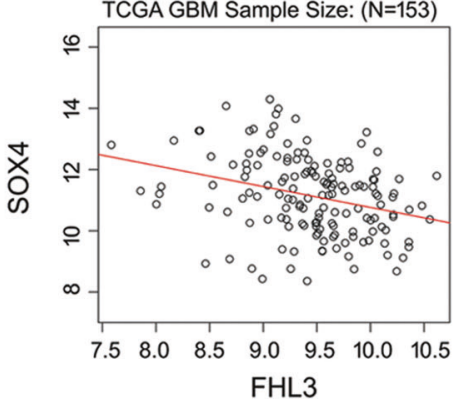

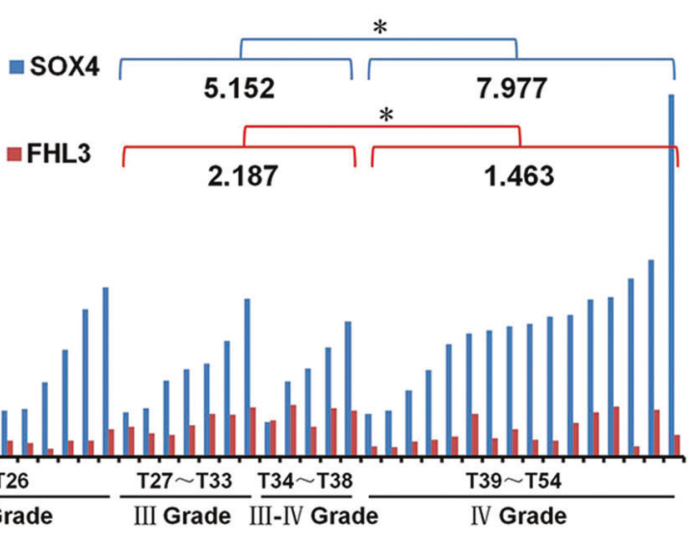

could regulate the activity of the SOX4 promoter using a reporter gene assay (Fig. 3a) with or without TGF- $\beta 1$. FHL3 overexpression decreased the activity of a $\mathrm{SOX} 4$ promoter reporter in a TGF- $\beta 1$-independent manner (Fig. $3 b$ ). To further confirm this result, we synthesized 2 siRNAs (siFHL3-1 and siFHL3-2) that specifically targeted FHL3, as previously reported $[12,14]$. Either 48 or $72 \mathrm{~h}$ after transfection in T98G glioma cells, knockdown of 
Fig. 3 FHL3 suppresses SOX4 transcriptional activity and TGF- $\beta$ responsive transcription in a TGF- $\beta 1$-independent manner. a Schematic diagram of the SOX4 promoter reporter construct. b, c T98G cells were co-transfected with an FHL3 overexpression construct (b) or FHL3 siRNAs (c), and either the dual luciferase reporter vector pEZX-PG04 or a SOX4 promoter reporter. Cells were treated with $(+)$ or without (-) TGF- $\beta 1$ and analyzed for Gaussia Luciferase (GLuc) and Secreted Alkaline Phosphatase (SEAP) activities, using the SEAP signal as an internal control. The normalized signals (ratio of GLuc to SEAP activities) are represented as the mean \pm SD of three independent experiments. $* P<0.05$ versus empty vector (b) or control siRNA (c) without TGF- $\beta 1 .{ }^{\#} P<0.05$ versus empty vector (b) or control siRNA (c) with TGF- $\beta 1$. d T98G cells were co-transfected with the TGF- $\beta$ signaling pathway reporter p3TP-Lux and either an FHL3 overexpression construct or empty vector. Cells were treated with $(+)$ or without (-) TGF- $\beta 1$ and analyzed for luciferase activity. Values are presented as the mean $\pm \mathrm{SD}$ of three independent experiments. $* P<$ 0.05 versus empty vector without TGF- $\beta 1$. ${ }^{\#} P<0.05$ versus empty vector with TGF- $\beta 1$. e Microarray results showing changes in the expression of TGF- $\beta$-responsive genes upon FHL3 overexpression. Red represents upregulated genes, while blue represents downregulated genes. f The correlation between FHL3 and SOX4 mRNA in TCGA GBM samples was analyzed on the LinkedOmics website using a Spearman correlation test. g Relative SOX4 and FHL3 protein levels in 13 grade II, 13 grade II-III, 7 grade III, 5 grade III-IV, and 16 grade IV glioma tissues compared with 7 normal brain tissue controls. Western blotting results were quantified using ImageJ software and are shown as the relative ratios of SOX $4 / \beta$-Actin or FHL3/ $\beta$-Actin protein levels (average values shown above the blots). $* P<0.05 . P$ values were generated using an unpaired $t$-test

endogenous FHL3 enhanced SOX4 promoter reporter activity (Fig. 3c). Both with and without TGF- $\beta 1$, FHL3 weakened the activity of p3TP-Lux (a TGF- $\beta 1$-responsive reporter) in glioma cells (Fig. 3d). Consistent with this result, FHL3 decreased Smad2/3 phosphorylation (Supplementary Figure 2a). siRNA-mediated knockdown of FHL3 increased phosphorylated Smad2/3 protein levels (Supplementary Figure $2 \mathrm{~b}$ ). In addition to SOX4, we also found that some other genes downstream of the TGF- $\beta 1$ signaling pathway (e.g., CDKN1A, ID2, FN1) are downregulated in FHL3 overexpression microarrays (Fig. 3e).

Once we confirmed that FHL3 expression is decreased in the glioma tissues, consistent with our previous report, and that FHL3 negatively regulated SOX4 RNA and protein levels, we expected to observe enhanced SOX4 expression in glioma tissues and cell lines. Congruent with previous reports $[15,16]$, the SOX4 mRNA and protein expression was increased in a total of 54 glioma tissue samples of different grades compared with 7 normal brain tissue control samples (Supplementary Figure 3a, b). Compared with 3 normal human astrocyte cell lines (HA, HAc, and HAsp), SOX4 expression was upregulated in 9 glioma cell lines (Supplementary Figure 3c, d). SOX4 was highly expressed in all FHL3-downregulated cell lines (Supplementary Figure 3d). Next, we used GEPIA to detect SOX4 expression levels in samples from The Cancer Genome Atlas (TCGA) and Genotype-Tissue Expression (GTEx) program [17].
Compared to normal tissues, SOX4 expression levels were significantly increased in GBM and low-grade glioma (LGG) patient samples (Supplementary Figure 3e). Using the LinkedOmics database [18], we identified a negative correlation between FHL3 and SOX4 RNA levels in the TCGA GBM samples $(n=153)$. (Spearman's correlation = $-0.3226, P=4.751$ e-05) (Fig. 3f). We also observed a strong inverse correlation between SOX4 and FHL3 protein levels in high-grade glioma samples (Fig. 3g).

\section{FHL3 decreases the self-renewal, not proliferation, of glioma stem cells}

Does FHL3 also inhibit the proliferation of glioma stem cells? To test this hypothesis, we used patient-derived glioma stem cells. These cells, called GSC2 cells, were cultured in serum-free neurobasal medium. In addition, we enriched two glioma stem-like cell lines from U87MG and U251 cells; these were strictly kept under nondifferentiating growth conditions and are referred to as U87MG stem-like cells (U87MG-SLC) and U251 stem-like cells (U251-SLC). All three cell lines were able to selfrenew, differentiate into multiple lineages and form brain tumors in nude mice [19]. Unexpectedly, though FHL3 overexpression inhibited proliferation of glioma cells, this effect was not observed in U251-SLCs. We even found that FHL3 promoted the growth of U251-SLCs after $48 \mathrm{~h}$ of transfection (Fig. 4a). However, in GSC2 cells, the cell growth curve was almost unaffected by FHL3 (Supplementary Figure 4a). Stable SOX4 overexpression and knockdown produced an increase and decrease, respectively, in the proliferation of GSC2 cells (Supplementary Figures 4b, c). Annexin V-fluorescein isothiocyanate (FITC)/phosphatidylinositol (PI) double staining was performed to detect apoptosis induced by FHL3 overexpression. Flow cytometric results showed an increase in the percentage of FITC- and PI-positive apoptotic cells from 24.8 to $42.3 \%$ in U251 cells; however, there was no difference in the percentage of apoptotic U251-SLCs (19.3\% $\pm 3.0 \%$ FITC- and PI-positive cells versus $21.9 \% \pm 6.7 \%, P$ $>0.05$ ) (Fig. 4b). Similarly, overexpression of FHL3 increased levels of cleaved caspase-3 and cleaved polyADP-ribose polymerase (PARP) in U251 cells but not in U251-SLCs (Fig. 4c).

As there are significant differences in epigenetic regulation between non-stem glioma cells and glioma stem cells [5], we repeated our ChIP-PCR experiment to detect FHL3 occupancy in the regions upstream of the DDIT3, $C A V 1$, and SOX4 genes in GSC2 cells. In GSCs, we found that FHL3 binding regions were lost upstream of DDIT3 and CAVI, however the binding sites upstream of SOX4 were retained (Fig. 4d). Further, we verified that Smad2/3 complexes were directly bound to the SOX4 promoter 
A

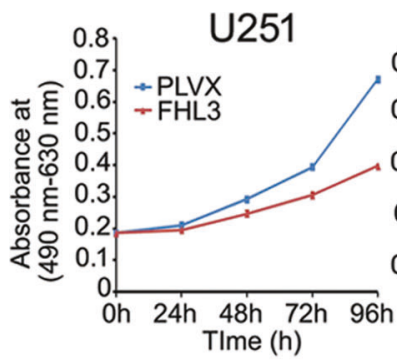

0.5. U251-SLC

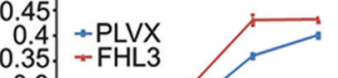

H

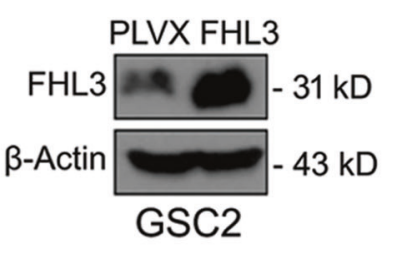

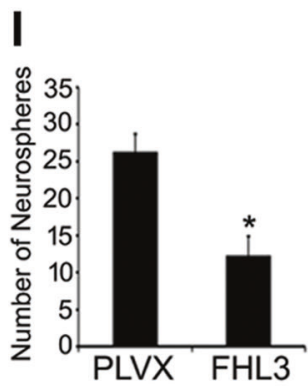

B

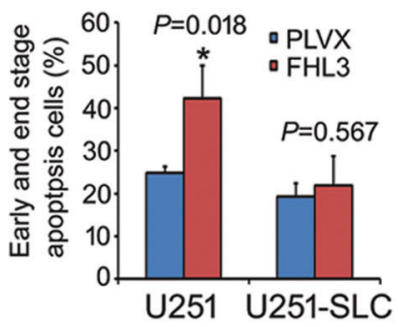

C

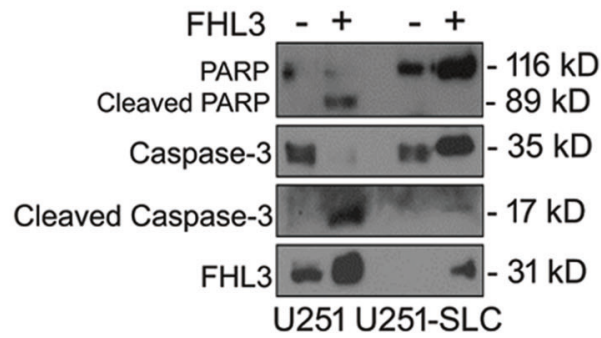

D
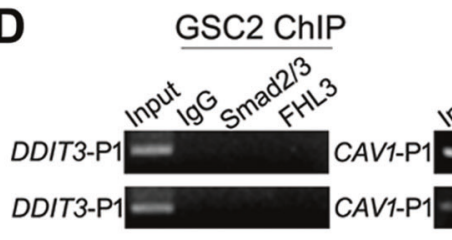

GSC2 ChIP

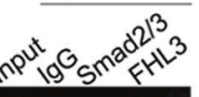

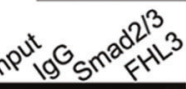

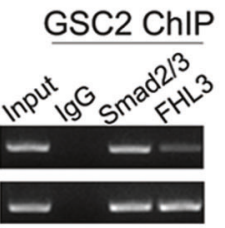

J

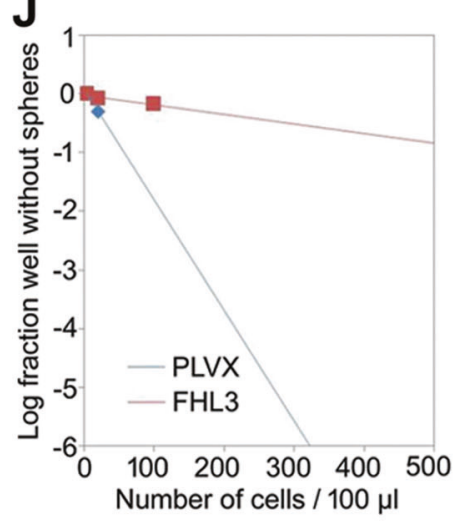

E

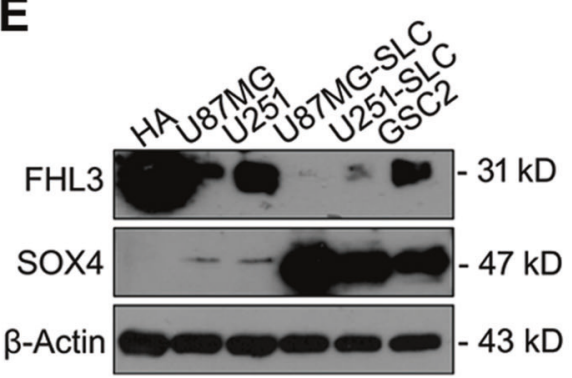

$\mathbf{F}$

$\mathrm{pH} \quad 7.4 \quad 6.8$

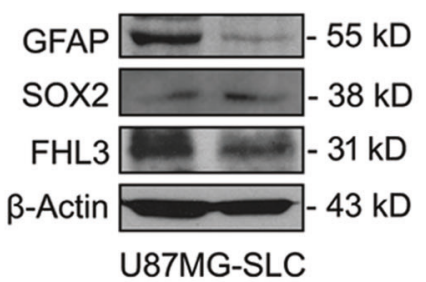

G

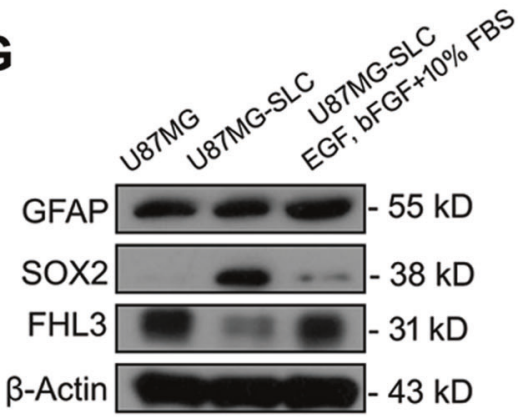

Fig. 4 FHL3 inhibits the self-renewal of glioma stem cells. a MTS assays demonstrating the growth of U251 (left) glioma cells and U251SLC (right) stem cell-like glioma cells after transfection with either an FHL3 overexpression plasmid or a control vector (PLVX). b Flow cytometry-based quantification of apoptosis by Annexin V/PI staining in U251 and U251-SLC cells $\sim 72 \mathrm{~h}$ following transfection. Early end stage apoptotic cell ratios were calculated and plotted on the histogram. c Representative western blot showing (cleaved) caspase-3 and its substrate, (cleaved) poly (ADP-ribose) polymerase (PARP) in transfected U251 and U251-SLC cells. d ChIP-PCR assays in GSC2 glioma stem cells. Soluble chromatin was prepared from GSC2 cells and subjected to immunoprecipitation with rabbit $\operatorname{IgG}$ (as a negative control) or the indicated antibodies. An anti-Smad2/3 antibody was used as a positive control for enrichment of the SOX4 promoter. PCR primers were designed to amplify the DDIT3, CAV1, or SOX4 promoter regions, as described previously. e Western blot analysis of FHL3 and SOX4 protein levels in HA normal human astrocytes, U87MG and U251 glioma cell lines, and U87MG-SLC, U251-SLC, and GSC2 stem cell-like glioma cells. f U87MG stem cell-like glioma cells were cultured in $\mathrm{pH} 7.4$ or $\mathrm{pH}$ 6.8 medium. Western blot showing GFAP, SOX2, and FHL3 protein levels under these $\mathrm{pH}$ conditions. $\mathrm{g}$ Western blot showing FHL3 protein levels during the differentiation of U87MG stem cell-like glioma cells. GFAP was used as a marker of cell differentiation. SOX2 was used as a marker of stemness. h Western blot analysis of FHL3 expression in GSC2 cells infected with PLVX (control) or FHL3-overexpressing lentivirus. i Sphere formation assay in GSC2 cells infected with PLVX (control) or FHL3-overexpressing lentivirus. Neurospheres (diameter $\geq$ $50 \mu \mathrm{m}$ ) were counted. j GSC2 self-renewal capacity was measured using a limiting dilution assay. Cells infected with PLVX lentivirus were used as controls. For each cell plating density, wells not containing spheres (diameter $\geq 50 \mu \mathrm{m}$ ) were quantified. Data are presented as the mean $\pm \mathrm{SD}$ of three independent experiments. ${ }^{*} P<0.05$ 
A

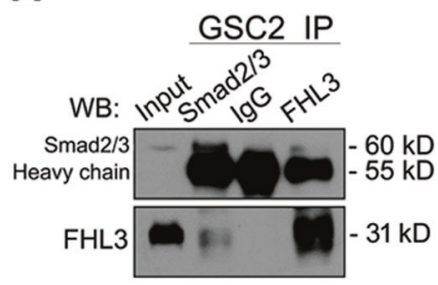

B

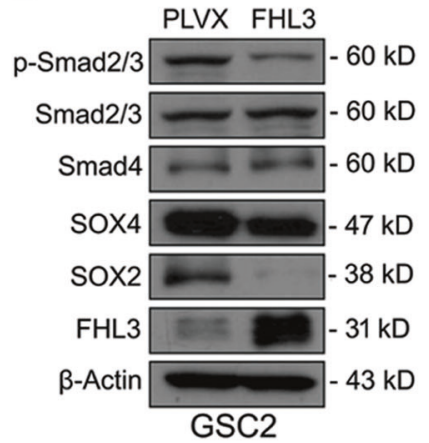

C

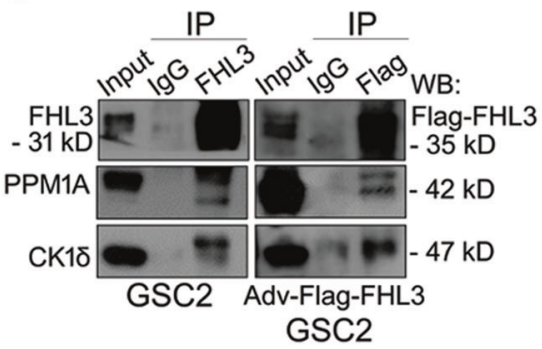

D

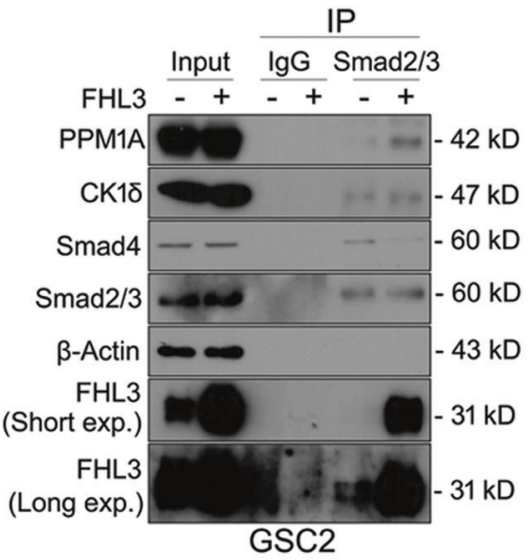

G

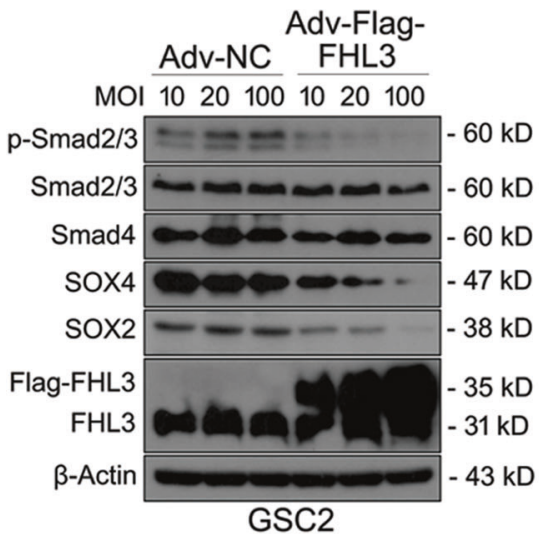

Fig. 5 FHL3 regulates Smad2/3 phosphorylation and suppresses the Smad2/3-SOX4-SOX2 axis. a For coimmunoprecipitation assays, GCS 2 cells were fractionated and proteins were immunoprecipitated using anti-Smad2/3 or anti-FHL3 antibodies or pre-immune control serum (IgG). Precipitates were analyzed by western blotting with the indicated antibodies. b Representative western blot showing levels of FHL3 and Smad2/3-SOX4-SOX2 axis-related proteins in GSC2 cells infected with PLVX or PLVX-FHL3 lentivirus. c Non-infected and Flag-FHL3 adenovirus-infected GSC2 cells were fractionated and proteins were immunoprecipitated with an anti-FHL3 antibody and an anti-Flag antibody, respectively. Anti-PPM1A and anti-CK1 $\delta$ antibodies were used to detect interactions with FHL3 protein by western blot. d GSC2 cells infected with PLVX (-) or PLVX-FHL3 $(+)$ lentivirus were immunoprecipitated with an anti-Smad2/3

$\mathbf{F}$

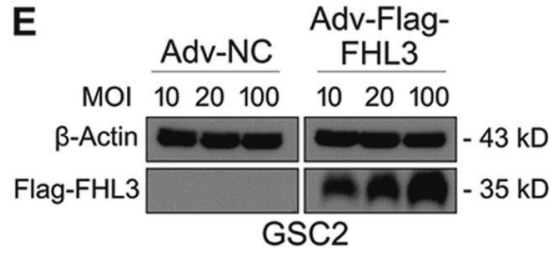

\begin{tabular}{|c|c|c|c|}
\hline & Input & $\lg G$ & Flag \\
\hline $\operatorname{Adv}-\mathrm{NC}(\mathrm{MOI})$ & & 20 & 20 \\
\hline $\mathrm{g}-\mathrm{FHL} 3(\mathrm{MOI})$ & 1020100 & 1020100 & 1020100 \\
\hline Sox4-P1 & & & $--\infty$ \\
\hline SOX4-P2 & ש- & & c \\
\hline
\end{tabular}

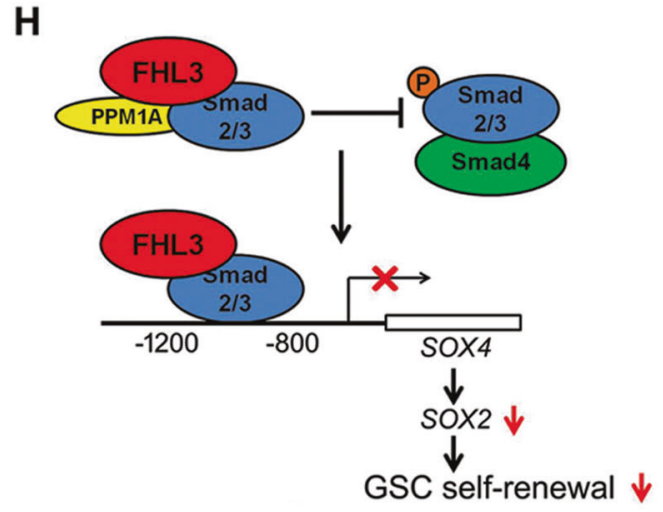

antibody or IgG. Precipitates were analyzed by western blotting with the indicated antibodies. e GSC2 cells were infected with adenovirus at 10, 20, and 100 multiplicities of infection (MOI). An anti-Flag antibody was used to detect increases in Flag-FHL3 levels. Adv-NC was used as a control. f ChIP-PCR assays in GSC2 cells infected with adenovirus at the indicated MOI. Soluble chromatin was subjected to immunoprecipitation with rabbit IgG or anti-Flag antibodies. Immunoprecipitated DNA was subjected to PCR to amplify the $S O X 4$ promoter region using the primers described previously. $g$ Representative western blot showing Smad2/3-SOX4-SOX2 axisrelated proteins and FHL3 protein levels in GSC2 cells infected with Adv-NC or Adv-Flag-FHL3 at the indicated MOI. h Proposed model of FHL3 modulation of the Smad2/3-SOX4-SOX2 axis 
A

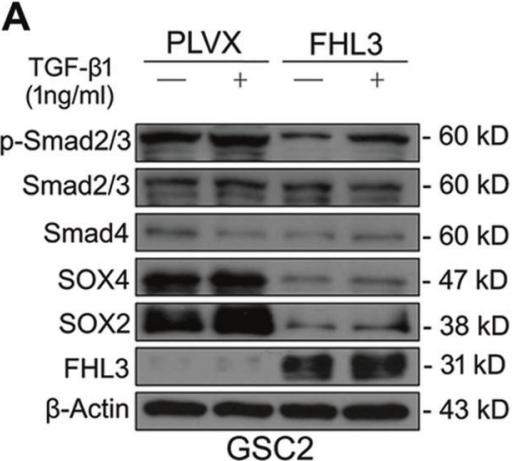

D

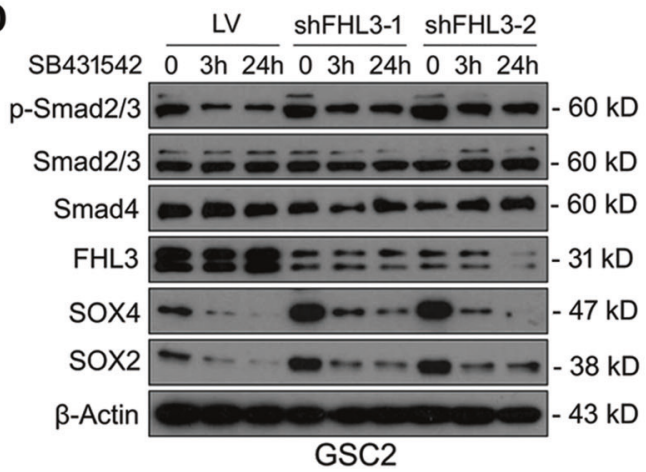

Fig. 6 FHL3 antagonizes TGF- $\beta 1$-mediated activation of the Smad2/ 3-SOX4-SOX2 axis. a Representative western blot showing Smad2/ 3-SOX4-SOX2 axis-related proteins and FHL3 protein levels in GSC2 cells infected with PLVX vector or PLVX-FHL3 lentivirus with or without $1 \mathrm{ng} / \mathrm{ml} \mathrm{TGF-} \beta 1$. b Sphere formation assay in GSC2 cells with or without $1 \mathrm{ng} / \mathrm{ml}$ TGF- $\beta 1$. c Limiting dilution neurosphere assay in GSC2 cells infected with PLVX vector or PLVX-FHL3 lentivirus with or without $1 \mathrm{ng} / \mathrm{ml}$ TGF- $\beta 1$. d GSC2 cells after

(Fig. 4d), which is in line with what has been previously reported in other GSCs [20]. To test whether FHL3 maintains its negative regulation of SOX4 in GSCs, we further examined their expression by western blot. The results showed that lower FHL3 protein levels correlated with higher SOX4 levels, an effect mainly observed in stem-like glioma cells (Fig. 4e).

Exposure to low $\mathrm{pH}$ promotes malignancy through the induction of a glioma stem cell phenotype [21]. U87MGSLCs were exposed to standard $\mathrm{pH}$ (7.4) or an acidic $\mathrm{pH}$ (6.8). Cells grown in low $\mathrm{pH}$ conditions displayed a decrease in FHL3 levels along with lower expression levels of the astrocyte marker GFAP and higher expression levels of the stemness marker SOX2 (Fig. 4f). We removed cytokines EGF and bFGF from the medium and added $10 \%$ serum to the medium for 8 days in order to induce U87MGSLC differentiation. During this differentiation process, FHL3 levels increased, whereas SOX2 levels decreased (Fig. 4g). FHL3-overexpressing glioma stem cells displayed less self-renewal capacity by sphere formation and limiting dilution assays (Fig. 4h-j), Similar results were obtained in U87MG-SLCs and U251-SLCs (Supplementary Figure 5a-

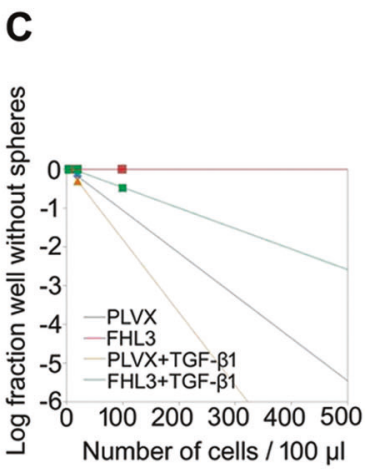

E

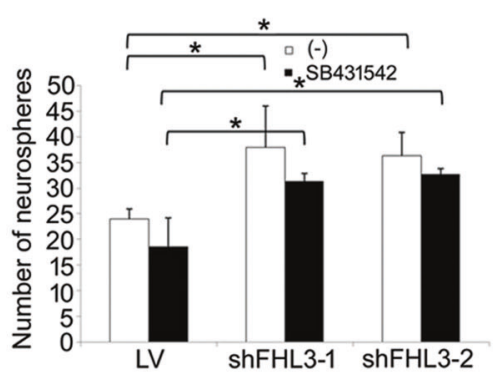

infection with either the control shRNA lentivirus (LV) or an shFHL3 lentivirus (shFHL3-1 and shFHL3-2) were cultured with a TGF- $\beta 1$ inhibitor (SB431542, $1 \mu \mathrm{M}$ ) for 0, 3, and $24 \mathrm{~h}$. Representative western blot showing Smad2/3-SOX4-SOX2 axis-related proteins and FHL3 protein levels in these stem cell-like glioma cells. e Sphere formation assay in infected GSC2 cells treated with a TGF- $\beta 1$ inhibitor (SB431542, $1 \mu \mathrm{M}$ ) for 0 and 7 days. Data are presented as the mean \pm SD of three independent experiments. $* P<0.05$

5b). Taken together, these data suggest that FHL3 does not alter glioma stem cell proliferation but rather affects the self-renewal capacity of GSCs.

\section{FHL3 suppresses the Smad2/3-SOX4-SOX2 axis through dephosphorylation of Smad2/3}

SOX4 is involved in the Smad2/3-SOX4-SOX2 axis in glioma-initiating cells [20]. Further, SOX2, a marker of stemness, is one of the core transcription factors that can reprogram differentiated glioblastoma cells to glioblastoma stem-like cells [22]. We were interested in whether FHL3 was also involved in this pathway and whether it regulated the stemness of glioma cells. We verified the FHL3-Smad2/ 3 protein interaction in GSC 2 cells by immunoprecipitation assay (Fig. 5a). We found that knockdown of either Smad2 or Smad3 led to a decline in Smad2 and Smad3 phosphorylation and the inhibition of SOX4 and SOX2 expression. Simultaneous knockdown of Smad2 and Smad3 more significantly inhibited the Smad2/3-SOX4-SOX2 axis but did not inhibit Smad4 (Supplementary Figure 5c). We next tested the ability of FHL3 to affect the Smad2/ 
A

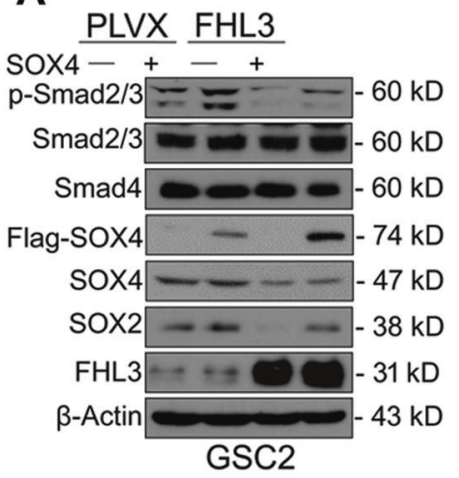

D

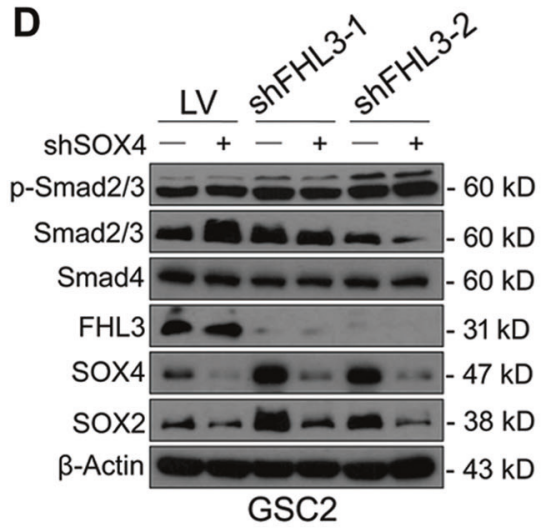

B

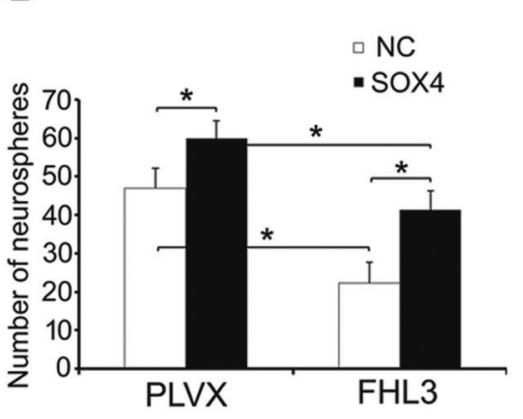

C

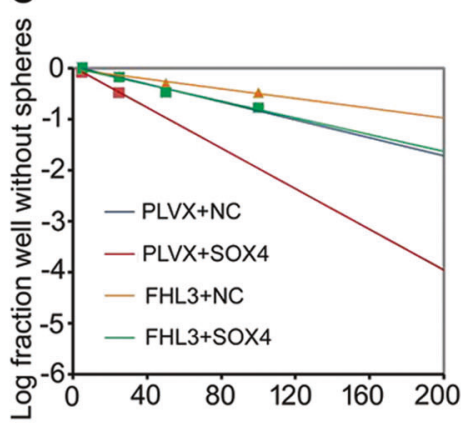

E

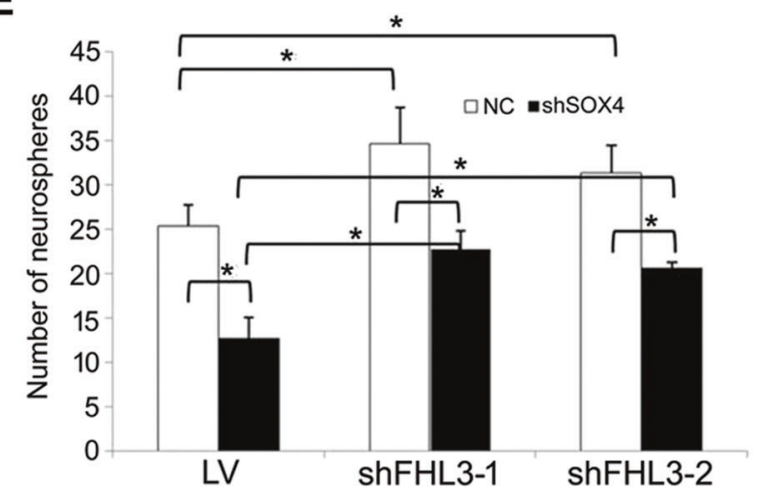

$\mathbf{F}$

\begin{tabular}{|c|c|c|c|c|}
\hline Cells & $-1-$ & $-150 \times 4$ & FHL3/- & FHL3/SOX4 \\
\hline 100,000 & $5(5)$ & $5(5)$ & $5(5)$ & $5(5)$ \\
\hline 50,000 & $4(5)$ & $3(5)$ & $0(5)$ & $3(5)$ \\
\hline 5,000 & $0(5)$ & $1(5)$ & $0(5)$ & $0(5)$ \\
\hline TIF & $1 / 31819$ & $1 / 36549$ & $1 / 96517$ & $1 / 43442$ \\
\hline$p$ & & 0.362 & & \\
\hline$p$ & & & 0.027 & \\
\hline$p$ & & & & 0.0171 \\
\hline
\end{tabular}

Fig. 7 SOX4 rescues FHL3-mediated inhibition of SOX2 (stemness marker) expression and sphere-forming ability in glioma stem cells. a Following infection with PLVX vector or PLVX-FHL3 lentivirus, GSC2 cells were re-infected with control or Flag-SOX4 lentivirus. Representative western blot showing expression of FHL3 and Smad2/3-SOX4-SOX2 axis-related proteins in the infected GSC2 cells. b Sphere formation assay in the infected GSC2 cells. c Limiting dilution neurosphere assay in the infected GSC2 cells. d Following infection with control shRNA lentivirus (LV) or shFHL3 lentivirus (shFHL3-1 and shFHL3-2), GSC2 cells were re-infected with control or shSOX4 lentivirus. Representative western blot showing expression levels of Smad2/3-SOX4-SOX2 axis-related

3-SOX4-SOX2 axis. Stable overexpression of FHL3 in GSC2 cells and U251-SLCs produced a marked decrease in phospho-Smad2/3, SOX4, and SOX2 (Fig. 5b, Supplementary Figure 5d). These observations suggest that FHL3 has an inhibitory effect on the Smad2/ 3-SOX4-SOX2 axis. proteins and FHL3 in the infected GSC2 cells. e Sphere formation assay in the shRNA lentivirus-infected GSC2 cells. f Limiting dilution assay showing rescue of GSC2 tumor-initiating capacity by SOX4 in vivo. Limiting dilutions of the infected GSC2 cells were subcutaneously implanted into nude mice. The table shows the number of mice that grew tumors at week 10 (out of a total of 5 mice per group). Tumor-initiating frequency (TIF) was calculated using ELDA software. $P<0.05$ was used as the significance threshold for comparisons between the FHL3-overexpression group and the control group as well as the FHL3 and SOX4 co-overexpression group and the FHL3-overexpression group. Data are presented as the mean \pm SD of three independent experiments. $* P<0.05$

How does FHL3 block Smad2/3 phosphorylation? One might envision that FHL3 directly competes with kinases for Smad2/3 protein binding or that FHL3 recruits phosphatases to the Smad2/3 protein complex. Previous studies have shown that CK1 $\delta$ directly phosphorylates Smad2/3 proteins [12] and that PPM1A dephosphorylates Smad2/3 
[23]. We used an immunoprecipitation assay to identify the association between FHL3 and both CK1 $\delta$ and PPM1A (Fig. 5c). Importantly, increasing the expression of FHL3 enhanced the binding of the Smad2/3 protein complex to PPM1A and reduced the formation of Smad2/3 and Smad4 complexes (Fig. 5d). We therefore postulate that FHL3 dephosphorylates Smad2/3 through an interaction with the Smad phosphatase PPM1A. Meanwhile, we also found that FHL3 overexpression increased SOX4 promoter occupancy (Fig. 5e, f) and decreased phosphorylated Smad2/3, SOX4 and SOX2 protein levels in a dose-dependent manner (Fig. 5g). Altogether, we concluded that FHL3 perturbs the Smad2/3-SOX4-SOX2 axis through interactions with dephosphorylated Smad2/3, forming a transcriptional repressor complex that binds to the SOX4 promoter and inhibits the expression of SOX4 and, subsequently, SOX2 (Fig. 5h).

\section{FHL3 antagonizes TGF- $\beta 1$-mediated activation of the Smad2/3-SOX4-SOX2 axis}

SOX4 expression can be directly induced by $\mathrm{Smad} 2 / 3$, which is downstream of TGF- $\beta 1$ signaling $[13,24]$. TGF- $\beta 1$ increased the protein expression levels of phosphorylated Smad2/3, SOX4, and SOX2 in GSC2 cells. However, even in the presence of TGF- $\beta 1$, this Smad2/3-SOX4-SOX2 axis was inhibited in cells stably overexpressing FHL3 (Fig. 6a). We observed similar results in glioma SLCs (Supplementary Figures 6a-b). FHL3 also antagonized TGF- $\beta 1$-mediated increases in the sphere-forming ability and self-renewal capacity of glioma stem cells (Fig. 6b, c). SB431542 can inhibit signaling through the TGF- $\beta 1$ receptor ALK5 [25]. We found that the Smad2/ 3-SOX4-SOX2 axis was suppressed by SB431542 after only a $3 \mathrm{~h}$ treatment in GSC2 cells and U251-SLCs (Fig. 6d, Supplementary Figure 6c). Inhibition of FHL3 increased the expression of phosphorylated Smad2/3, SOX4, and SOX2 in untreated GSCs and partly restored the activity of the Smad2/3-SOX4-SOX2 axis in the presence of SB431542 (Fig. 6d, Supplementary Figure 6c). Stable knockdown of FHL3 also promoted sphere formation in GSC2 cells and U251-SLCs both with and without SB431542 (Fig. 6e, Supplementary Figure 6d). These results support our hypothesis that FHL3 suppresses the maintenance of stemness by downregulating the Smad2/3-SOX4-SOX2 axis in GSCs.

\section{SOX4 rescues FHL3-mediated maintenance of stemness in vitro and in vivo}

To further test the above hypothesis, we performed cooverexpression or co-knockdown of FHL3 and SOX4 for rescue studies. Western blot analyses revealed that SOX4 rescued SOX2 downregulation due to overexpression of FHL3 (Fig. 7a). Utilizing sphere formation and limiting dilution assays, we also observed that SOX4 overexpression reversed the effects of FHL3 on the sphere-forming ability and self-renewal capacity of GSCs (Fig. 7b, c). Similar rescue results were observed in U251-SLCs (Supplementary Figure 7a, b). Knockdown of SOX4 inhibited SOX2 expression but had no obvious effect on Smad2/3 phosphorylation. Further, decreases in SOX4 expression reduced the FHL3 knockdown-mediated increase in SOX2 expression (Fig. 7d). GSCs infected with shSOX4 lentivirus formed glioma spheres with lower efficiency. In contrast to knockdown of FHL3 alone, a decreased number of glioma spheres were formed by GSC2 cells with co-knockdown of FHL3 and SOX4, suggesting impaired self-renewal (Fig. 7e). In U251-SLCs, similar rescue effects were shown (Supplementary Figure 7c, d). To study the role of FHL3-mediated regulation of SOX4 in the maintenance of stemness in vivo, we examined the effects of FHL3 and SOX4 on GSC tumorigenicity in nude mice. Accordingly, control GSC2 cells as well as cells overexpressing SOX4 or FHL3 alone or co-overexpressing SOX4 and FHL3 were injected into immunodeficient mice in limiting dilutions. Cells overexpressing FHL3 alone displayed a threefold reduction in tumor-initiating frequency (TIF), confirming that FHL3 reduces the maintenance of tumor cell stemness (Fig. 7f). Although there was no significant change in the TIF of cells overexpressing SOX4 alone, the overexpression of SOX4 could restore the TIF of FHL3-overexpressing cells by more than twofold (Fig. 7f). Altogether, these data indicate that FHL3 inhibits GSC stemness by reducing the expression of SOX4 in vitro and in vivo.

\section{Discussion}

In this study, we tested 11 candidate target genes regulated by FHL3 in non-stem glioma cells. Of these, SOX4, CAV1, and DDIT3 were confirmed by ChIP-PCR and western blotting. Interestingly, unlike in non-stem glioma cells, CAVI and DDIT3 were unable to cooperate with FHL3 in GSCs. Further, we found that FHL3 could not impede GSC growth as in non-stem glioma cells. Although SOX4 can affect GSC proliferation, its influence is not as pronounced. Our results suggest that FHL3 modulates cell growth in non-glioma cells but controls self-renewal in GSCs. This could be due to the fact that GSCs display more "stemness" features but a limited proliferative ability. Many proteins have been reported to perform distinct functions in stem and non-stem cells. For example, Sema3C depletion induces apoptosis in GSCs but not in non-stem glioma cells [26]. The variable responses to FHL3 in different cells indicate that it has a suppressive role in glioma initiation. 
TGF- $\beta 1$ signaling induces SOX 4 expression via $\mathrm{Smad} 2 / 3$ [20]. Then, SOX4 induces the expression of the stem cell marker SOX 2 through cooperative binding with Oct 4 at the SOX2 promoter. This process is critical for TGF- $\beta 1$ mediated maintenance of stemness in glioma stem cells $[27,28]$. We demonstrated for the first time that SOX4 is regulated by FHL3 at the transcriptional level and that FHL3 suppresses the stemness of glioma stem cells by inhibiting the Smad2/3-SOX4-SOX2 axis. The stemness marker SOX2 is increased in glioma stem cells and may drive the oncogenic transformation of mouse astrocytes [29].

FHL3 can act as either a transcriptional co-activator or co-repressor. Ding et al. demonstrated that the FHL3-Smad interaction enhances p3TP-Lux reporter activity in hepatocellular carcinoma cells, while we observed that a transcription complex containing similar components abates p3TP-Lux and SOX4 promoter reporter activity in glioma cells. In accordance with previous results, we also found that FHL3 can interact with CK1 $\delta$. Further, we detected an interaction between FHL3 and PPM1A. PPM1A functions as a Smad phosphatase to enable the nuclear export of Smad2/3 [30] and also has tumor suppressor-like activity [31]. Our data suggest that the mechanism of FHL3mediated regulation of $\operatorname{Smad} 2 / 3$ phosphorylation has two sides. We believe that this is due to tissue specificity and tumor specificity in human cancers.

SOX4 also has disparate roles as either a tumor suppressor or an oncogene and affects key processes related to tumor biology including cell proliferation, apoptosis, migration, metastasis, and cancer stemness [24]. In primary hepatocellular carcinoma, $\mathrm{SOX} 4$ acts as a tumor suppressor through interactions with the DNA-damage pathway [32]. In addition to glioma, SOX4 has been reported to contribute to cell survival in other cancers, such as prostate cancer [33] and adenoid cystic carcinoma [34]. SOX4 deletion could contribute to a strong resistance to tumor development [35]. SOX4 was previously reported to inhibit glioma cell growth and induce cell cycle arrest [36]; however, our results showed that SOX4 can drive glioma progression.

Perturbations in transforming growth factor- $\beta$ (TGF- $\beta$ ) signaling are central to tumorigenesis and tumor progression through effects on cellular processes [37]. In highgrade glioma, TGF- $\beta$-Smad $2 / 3$ activity and stem cell markers are frequently upregulated and correlate with worse patient survival [38, 39]. In GSCs, the TGF $\beta$ signaling axis is activated by the $\alpha v \beta 8$ integrin to drive tumor initiation and progression [40].TGF- $\beta$ signaling has crucial roles in the maintenance of self-renewal and tumorigenicity in glioma stem cells through the induction of LIF, SOX4, and SOX2 [20, 41]. Accordingly, we found that FHL3 could antagonize TGF- $\beta-\operatorname{Smad} 2 / 3$ activity by inhibiting the expression of SOX4-SOX2. What is particularly noteworthy is that combined treatment with TGF- $\beta$ inhibitors and radiation can improve therapeutic responses in patients with GBM $[42,43]$. We believe that FHL3 may be a key gene involved in glioma susceptibility to radiotherapy. Altogether, our results shed light on some crucial functions of the FHL3 protein in glioma initiation and progression.

\section{Materials and Methods}

\section{Tissue samples}

Normal brain and glioma tissues were obtained from the human brain bank of the Institute of Basic Medical Sciences, Chinese Academy of Medical Sciences and the Department of Neurosurgery, Beijing Tiantan Hospital. All samples were classified according to the third edition of the histological grading of tumors of the nervous system published by the WHO in 2000. Informed consent for the use of samples was obtained from all patients before surgery and approval was obtained from the Medical Ethics Committee of the Beijing Tiantan Hospital (Beijing, China).

\section{Cell lines and cell culture}

The human glioma cell lines T98G, U87MG, and A172 were purchased from ATCC and cultured according to the guidelines recommended by the ATCC. The U251, Hs683, SHG-44, SF126, SF763, and SF767 cell lines (from the Cell Center of Peking Union Medical College) were cultured in minimum essential medium and Iscove's modified Dulbecco's medium supplemented with $10 \%$ FBS. All cells were maintained at $37^{\circ} \mathrm{C}$ with $5 \% \mathrm{CO}_{2}$. The $\mathrm{HA}, \mathrm{HAc}$ and HAsp cell lines were purchased from ScienCell Research Laboratories and cultured in astrocyte medium. The isolation, culture, and identification of GSC2 cells and the enrichment of glioma stem-like cells (U87MG-SLCs and U251-SLCs) were performed as described previously [19]. GSCs and SLCs were digested with $0.05 \%$ trypsin (Life Science), washed twice with PBS and subsequently cultured in serum-free stem cell medium (Neuralbasal (Life science, USA) containing $20 \mathrm{ng} / \mathrm{ml}$ basic fibroblast growth factor (bFGF) (Peprotech, USA), $20 \mathrm{ng} / \mathrm{ml}$ epidermal growth factor (EGF) (Peprotech), $10 \mu \mathrm{g} / \mathrm{ml}$ heparin (Sigma), 2\% B27 Supplement (Life Science), and $2 \mathrm{mmol} / \mathrm{l} \mathrm{L-glutamine} \mathrm{(Life}$ Science)).

\section{Plasmids, siRNAs, and transfection}

The FHL3 expression construct and the p3TP-Lux reporter were gifts from Qinong Ye of the Beijing Institute of Biotechnology (Beijing, China). The CAV1 and DDIT3 coding 
regions were amplified by PCR and inserted into the pcDNA6.0-Flag-tagged vector. The FHL3 siRNA (shRNAs) sequences for FHL3 have been described previously $[12,14]$. The SOX4 shRNA sequences were as described previously [34]. All of the plasmids were purified using the EndoFree Plasmid Maxi Kit (QIAGEN) and transfected into T98G cells using FuGENE6 (Promega). siRNAs were synthesized by the Shanghai GenePharma Company and transfected into glioma cell lines using INTERFERin reagent (Ployplus) at a final concentration of $50 \mathrm{nM}$.

\section{Lentiviral and adenoviral production and infection}

Construction of the PLVX plasmids and packaging of the FHL3 overexpression lentiviruses were performed at Shanghai Chempartner. Construction of the SOX4 and FHL3 knockdown plasmids and the construction and packaging of the SOX4 overexpression lentiviruses were performed at GeneChem. Flag-FHL3 overexpression adenoviruses were also synthesized at GeneChem. Infected cells were selected with puromycin $(2 \mu \mathrm{g} / \mathrm{ml})$ for 14 days. SOX4 overexpression lentiviruses expressed GFP and a fusion-Flag-tagged protein. The FHL3-knockdown lentivirus expressed GFP, while the SOX4-knockdown lentivirus expressed red fluorescent protein. These fluorescent markers were used to select stably-infected cells. Lentiviral particles were added to the cells with $5 \mu \mathrm{g} / \mathrm{ml}$ polybrene to increase the efficiency of virus infection.

\section{Immunoprecipitation and western blot analysis}

Whole cells or tissues were collected and homogenized with TNTE lysis buffer containing protease inhibitors. After incubation for $30 \mathrm{~min}$ on ice, the homogenate was centrifuged at $18,000 \times g$ for $20 \mathrm{~min}$. The supernatants were stored at $-80{ }^{\circ} \mathrm{C}$ until the immunoprecipitation assay was performed. For the immunoprecipitation assay, equal amounts (1200-1500 $\mu \mathrm{g})$ of cell lysates were incubated with $1 / 100(\mathrm{wt} / \mathrm{wt})$ of anti-Smad2/3 antibody (Cell Signaling Technology, 3102), anti-FHL3 antibody (Proteintech, 11028-2-AP), or anti-Flag antibody (Sigma, USA, F7425) overnight at $4{ }^{\circ} \mathrm{C}$. Subsequently, the lysates were incubated with Protein A Sepharose (GE Healthcare, UK) while rotating for $3 \mathrm{~h}$ at $4{ }^{\circ} \mathrm{C}$. Immunoprecipitates were washed four times with lysis buffer, eluted in $20 \mu \mathrm{l}$ of $6 \times$ loading buffer at $98^{\circ} \mathrm{C}$ and analyzed by western blotting. For western blot analyses, the protein samples were separated on a $10 \%$ SDS-PAGE gel, transferred to a nitrocellulose membrane (Amersham, USA) and blotted with anti-FHL3 (Proteintech, 11028-2-AP), anti-Smad2/3 (Cell Signaling Technology, 3102), anti-Smad4 (Santa Cruz, sc7154), antip-Smad2/3 (Santa Cruz, sc11769), anti-p-Smad2 (Bioworld,
BS4172), anti-p-Smad3 (Bioworld, BS64037), anti-CK1ס (Bioworld, BS5640), anti-PPM1A (Bioworld, BS8366), anti-Caspase-3 (Cell Signaling Technology, 9665), anticleaved Caspase-3 (Cell Signaling Technology, 9661), antiPARP (Cell Signaling Technology, 9542), anti-SOX4 (Santa Cruz, sc20090), anti-SOX2 (Abcam, ab97959), anti-GFAP (Covance, PRB-571C), anti-CAV1 (Santa Cruz, sc894), anti-DDIT3 (Santa Cruz, sc7351), anti-Flag tag (Sigma, USA, F7425), or anti- $\beta$-actin(Sigma, USA, A5441) antibodies.

\section{Real-time PCR}

Total RNA was purified using Trizol reagent (Invitrogen). Total RNA was used for cDNA synthesis with TransScript First-Strand cDNA Synthesis SuperMix (TransGen). Realtime PCR assays were performed with SYBR Premix EX $\mathrm{Taq}(\mathrm{TaKaRa})$. Ct values of target genes were normalized to those of the GAPDH gene. The $\Delta \Delta \mathrm{Ct}$ method was used to determine the relative expression levels of the target genes. All samples were run in triplicate in each experiment. Each assay was repeated three times. The sequences of the primers used are listed in Supplementary Table S1.

\section{Microarray analysis}

Total RNA was extracted from approximately $10^{6}$ T98G cells transfected with vector or FHL3 using Trizol reagent (Invitrogen). RNA integrity was measured using an Agilent Bioanalyzer 2100 instrument (Agilent Technologies, Santa Clara, CA). Gene expression microarrays, including three technical replicates of FHL3 overexpression versus the empty vector, were performed at the ShanghaiBio Corporation. We conservatively established a threshold of at least a twofold difference, with an FDR-adjusted $P$ value of $<0.05$. Biological functions (gene ontology) of the FHL3regulated genes identified by microarray analysis were determined using the web-based SBC analysis system.

\section{Dual luminescence promoter reporter assay}

These assays were carried out using GeneCopoeia GLucONTM Promoter Reporter Clones containing Gaussia Luciferase (GLuc) and Secreted Alkaline Phosphatase (SEAP). The SOX4 promoter clone contains a $1473 \mathrm{bp}$ insert, corresponding to the $5^{\prime}$-flanking sequence located approximately $1247 \mathrm{bp}$ upstream of the transcription initiation site and $225 \mathrm{bp}$ downstream of the transcription initiation site. This insert was placed upstream of the GLuc reporter gene. Both GLuc and SEAP are secreted reporter proteins. Samples can be easily obtained from the cell culture medium. At $48-72 \mathrm{~h}$ after transfection, the cell culture medium was gently collected for GLuc and SEAP 
luminescent assays. Using the SEAP signal as an internal control, signals were normalized (ratio of GLuc and SEAP activities) to eliminate the impact of transfection efficiency variations.

\section{ChIP-PCR}

For ChIP assays, approximately $1 \times 10^{7}$ glioma cells were harvested in medium and fixed with $1 \%$ formaldehyde. Glycine solution was added at a final concentration of 0.125 $M$ to quench unreacted formaldehyde. Fixed cells were collected by spinning at $700 \times g$ for $5 \mathrm{~min}$. Five micrograms of anti-FHL3 (Santa Cruz) or anti-Flag antibody (Sigma) or the isotype-control antibody (rabbit IgG, Zhongshan) was used in the assay. ChIP experiments were performed using the Magna ChIPTM A/G Chromatin Immunoprecipitation Kit (Invitrogen) according to the manufacturer's protocol. The resulting ChIP products were used for PCR. The PCR primers are listed in Supplementary Table S2.

\section{Cell proliferation and apoptosis analysis}

The MTS assay (Promega, USA) was used to evaluate the effects of FHL3, CAV1, DDIT3, and SOX4 on the proliferation of non-stem glioma cells and GSCs. Cells were seeded at a density of 3000 cells per well and $100 \mu$ of medium per well in 96-well plates. Twenty microliters of MTS $(2 \mathrm{mg} / \mathrm{ml}$ DPBS)/PMS (0.92 mg/ml DPBS) detection solution was added to each well and incubated for $2 \mathrm{~h}$. Absorbance was measured-with a test wavelength of 490 $\mathrm{nm}$ and a reference wavelength of $630 \mathrm{~nm}$ - to obtain sample signal. Cell apoptosis was assessed using the Annexin V-FITC/PI Detection Kit I (BD Pharmingen) according to the manufacturer's instructions.

\section{Neurosphere-formation and limiting dilution assays}

The neurosphere-formation and limiting dilution assays were performed as described previously [19]. In brief, sphere-forming GSCs or SLCs were dissociated into single cells. Cells were diluted to $1000-5000$ cells $/ \mathrm{ml}$, and $100 \mu \mathrm{l}$ of the cell solution was added into each well of a 96-well plate. Cells were allowed to grow for 6-8 days, with $50 \mu \mathrm{l}$ of fresh medium added at day 3 or 4 . Then, the number of neurospheres in each well $\geq 50 \mu \mathrm{m}$ in diameter were counted under a microscope. In the in vitro limiting dilution assays, sphere cells were dissociated into a single-cell suspension and plated in 96-well plates in $100 \mu$ l of culture medium. Final cell densities ranged from 500 to 3 cells per well in a volume of $100 \mu \mathrm{l}$ per well. Fresh medium was added every 4 days. After 12 days, the percentage of wells not containing spheres (diameter $\geq 50 \mu \mathrm{m}$ ) was calculated for each cell plating density.

\section{In vivo limiting dilution assay}

Four- to five-week-old male BALB/c athymic nu/nu mice were randomly divided into four groups with 15 mice in each group, as described in Fig. 7f. Serial dilutions of GSC2 cells $\left(5 \times 10^{3}, 5 \times 10^{4}, 1 \times 10^{5}\right)$ were subcutaneously injected in $0.1 \mathrm{ml}$ of PBS into replicates of 5 mice. After 10 weeks, the number of mice with tumors in each group was quantified. TIF was calculated using ELDA Software. All animal studies were approved by the IACUC of the Center for Experimental Animal Research (China), and all animal experiments were performed in accordance with institutional guidelines and abided by the declaration of ethical approval for experiments.

\section{Statistical analysis}

Data are presented as the mean \pm standard deviation of at least three independent experiments. Comparisons were determined using a paired or unpaired two-tailed Student's $t$-test using SPSS 17.0 software, and a $P$ value of $<0.05$ was considered statistically significant $(*)$. The investigators were not blinded to allocation during experimentation and outcome assessment. No samples or animals were excluded from the analyses.

\section{Accession numbers}

The accession number for the microarray data reported in this paper is NCBI GEO: GSE109988.

\begin{abstract}
Acknowledgements This study was supported by the following grants-The National Key Research and Development Program of China (2016YFC0902502, 2016YFA0100702), and National Sciences Foundation of China (31671316, 31670789), CAMS Innovation Fund for Medical Sciences (CIFMS, 2016-I2M-1-001, 2016-I2M-2-001, 2016-I2M-1-004，2017-I2M-2-004，2017-I2M-3-010，2017-I2M-1004), Peking Union Medical College Youth Fund, and the Fundamental Research Funds for the Central Universities (3332013052, 3332014006).
\end{abstract}

\section{Compliance with ethical standards}

Conflict of interest The authors declare that they have no conflict of interest.

\section{References}

1. Ostrom QT, Gittleman H, Fulop J, Liu M, Blanda R, Kromer C, et al. CBTRUS statistical report: primary brain and central nervous system tumors diagnosed in the United States in 2008-2012. Neuro Oncol. 2015;17:iv1-62.

2. Vescovi AL, Galli R, Reynolds BA. Brain tumour stem cells. Nat Rev Cancer. 2006;6:425-36.

3. Chen J, Li Y, Yu TS, McKay RM, Burns DK, Kernie SG, et al. A restricted cell population propagates glioblastoma growth after chemotherapy. Nature. 2012;488:522-6. 
4. Godek KM, Venere M, Wu Q, Mills KD, Hickey WF, Rich JN, et al. Chromosomal instability affects the tumorigenicity of glioblastoma tumor-initiating cells. Cancer Discov. 2016;6:532-45.

5. Liau BB, Sievers C, Donohue LK, Gillespie SM, Flavahan WA, Miller TE, et al. Adaptive chromatin remodeling drives glioblastoma stem cell plasticity and drug tolerance. Cell Stem Cell. 2017;20:233-46.

6. El-Habr EA, Dubois LG, Burel-Vandenbos F, Bogeas A, Lipecka $\mathrm{J}$, Turchi L, et al. A driver role for GABA metabolism in controlling stem and proliferative cell state through GHB production in glioma. Acta Neuropathol. 2017;133:645-60.

7. Han W, Xin Z, Zhao Z, Bao W, Lin X, Yin B, et al. RNA-binding protein PCBP2 modulates glioma growth by regulating FHL3. J Clin Invest. 2013;123:2103-18.

8. Fimia GM, De Cesare D, Sassone-Corsi P. A family of LIM-only transcriptional coactivators: tissue-specific expression and selective activation of CREB and CREM. Mol Cell Biol. 2000;20:8613-22.

9. Turner J, Nicholas H, Bishop D, Matthews JM, Crossley M. The LIM protein FHL3 binds basic Krüppel-like factor/Krüppel-like factor 3 and its co-repressor C-terminal-binding protein 2. J Biol Chem. 2003;278:12786-95.

10. Takahashi K, Matsumoto C, Ra C. FHL3 negatively regulates human high-affinity $\mathrm{IgE}$ receptor beta-chain gene expression by acting as a transcriptional co-repressor of MZF-1. Biochem J. 2005;386:191-200.

11. Cottle DL, McGrath MJ, Cowling BS, Coghill ID, Brown S, Mitchell CA. FHL3 binds MyoD and negatively regulates myotube formation. J Cell Sci. 2007;120:1423-35.

12. Ding L, Wang Z, Yan J, Yang X, Liu A, Qiu W, et al. Human four-and-a-half LIM family members suppress tumor cell growth through a TGF-beta-like signaling pathway. J Clin Invest. 2009;119:349-61.

13. Kuwahara M, Yamashita M, Shinoda K, Tofukuji S, Onodera A, Shinnakasu R, et al. The transcription factor Sox 4 is a downstream target of signaling by the cytokine TGF- $\beta$ and suppresses $\mathrm{T}(\mathrm{H}) 2$ differentiation. Nat Immunol. 2012;13:778-86.

14. Hubbi ME, Gilkes DM, Baek JH, Semenza GL. Four-and-a-half LIM domain proteins inhibit transactivation by hypoxia-inducible factor 1. J Biol Chem. 2012;28:6139-49.

15. Lin B, Madan A, Yoon JG, Fang X, Yan X, Kim TK, et al. Massively parallel signature sequencing and bioinformatics analysis identifies up-regulation of TGFBI and SOX4 in human glioblastoma. PLoS ONE. 2010;5:e10210.

16. Li L, Li Q, Chen X, Xu M, Li X, Nie L, et al. SOX4 is overexpressed in diffusely infiltrating astrocytoma and confers poor prognosis. Neuropathology. 2015;35:510-7.

17. Tang Z, Li C, Kang B, Gao G, Li C, Zhang Z. GEPIA: a web server for cancer and normal gene expression profiling and interactive analyses. Nucleic Acids Res. 2017;45:W98-102.

18. Vasaikar SV, Straub P, Wang J, Zhang B. LinkedOmics: analyzing multi-omics data within and across 32 cancer types. Nucleic Acids Res. 2018;46:D956-63.

19. Hu PS, Xia QS, Wu F, Li DK, Qi YJ, Hu Y, et al. NSPc1 promotes cancer stem cell self-renewal by repressing the synthesis of all-trans retinoic acid via targeting RDH16 in malignant glioma. Oncogene. 2017;36:4706-18.

20. Ikushima H, Todo T, Ino Y, Takahashi M, Miyazawa K, Miyazono K. Autocrine TGF-beta signaling maintains tumorigenicity of glioma-initiating cells through Sry-related HMG-box factors. Cell Stem Cell. 2009;5:504-14.

21. Hjelmeland AB, Wu Q, Heddleston JM, Choudhary GS, MacSwords J, Lathia JD, et al. Acidic stress promotes a glioma stem cell phenotype. Cell Death Differ. 2011;18:829-40.

22. Suvà $\mathrm{ML}$, Rheinbay $\mathrm{E}$, Gillespie $\mathrm{SM}$, Patel $\mathrm{AP}$, Wakimoto $\mathrm{H}$, Rabkin SD, et al. Reconstructing and reprogramming the tumor- propagating potential of glioblastoma stem-like cells. Cell. 2014;157:580-94.

23. Lin X, Duan X, Liang YY, Su Y, Wrighton KH, Long J, et al. PPM1A functions as a Smad phosphatase to terminate TGFbeta signaling. Cell. 2006;125:915-28.

24. Vervoort SJ, van Boxtel R, Coffer PJ. The role of SRY-related HMG box transcription factor 4 (SOX4) in tumorigenesis and metastasis: friend or foe? Oncogene. 2013;32:3397-409.

25. Inman GJ, Nicolás FJ, Callahan JF, Harling JD, Gaster LM, Reith $\mathrm{AD}$, et al. SB-431542 is a potent and specific inhibitor of transforming growth factor-beta superfamily type I activin receptor-like kinase (ALK) receptors ALK4, ALK5, and ALK7. Mol Pharmacol. 2002;62:65-74.

26. Man J, Shoemake J, Zhou W, Fang X, Wu Q, Rizzo A, et al. Sema3C promotes the survival and tumorigenicity of glioma stem cells through Rac1 activation. Cell Rep. 2014;9:1812-26.

27. Ikushima H, Todo T, Ino Y, Takahashi M, Saito N, Miyazawa K, et al. Glioma-initiating cells retain their tumorigenicity through integration of the Sox axis and Oct4 protein. J Biol Chem. 2011;286:41434-41.

28. Ying Z, Li Y, Wu J, Zhu X, Yang Y, Tian H, et al. Loss of miR204 expression enhances glioma migration and stem cell-like phenotype. Cancer Res. 2013;73:990-9.

29. Singh DK, Kollipara RK, Vemireddy V, Yang XL, Sun Y, Regmi $\mathrm{N}$, et al. Oncogenes activate an autonomous transcriptional regulatory circuit that drives glioblastoma. Cell Rep. 2017;18:961-76.

30. Dai F, Shen T, Li Z, Lin X, Feng XH. PPM1A dephosphorylates RanBP3 to enable efficient nuclear export of Smad2 and Smad3. EMBO Rep. 2011;12:1175-81.

31. Lu X, An H, Jin R, Zou M, Guo Y, Su PF, et al. PPM1A is a RelA phosphatase with tumor suppressor-like activity. Oncogene. 2014;33:2918-27.

32. Hur W, Rhim H, Jung CK, Kim JD, Bae SH, Jang JW, et al. SOX4 overexpression regulates the p53-mediated apoptosis in hepatocellular carcinoma: clinical implication and functional analysis in vitro. Carcinogenesis. 2010;31:1298-307.

33. Liu P, Ramachandran S, Ali Seyed M, Scharer CD, Laycock N, Dalton WB, et al. Sex-determining region $\mathrm{Y}$ box 4 is a transforming oncogene in human prostate cancer cells. Cancer Res. 2006;66:4011-9.

34. Pramoonjago P, Baras AS, Moskaluk CA. Knockdown of Sox4 expression by RNAi induces apoptosis in ACC3 cells. Oncogene. 2006;25:5626-39.

35. Foronda M, Martínez P, Schoeftner S, Gómez-López G, Schneider R, Flores JM, et al. Sox4 links tumor suppression to accelerated aging in mice by modulating stem cell activation. Cell Rep. 2014;8:487-500.

36. Zhang J, Jiang H, Shao J, Mao R, Liu J, Ma Y, et al. SOX4 inhibits GBM cell growth and induces G0/G1 cell cycle arrest through Akt-p53 axis. BMC Neurol. 2014;14:207.

37. Ikushima $H$, Miyazono K. TGFbeta signalling: a complex web in cancer progression. Nat Rev Cancer. 2010;10:415-24.

38. Bruna A, Darken RS, Rojo F, Ocaña A, Peñuelas S, Arias A, et al. High TGFbeta-Smad activity confers poor prognosis in glioma patients and promotes cell proliferation depending on the methylation of the PDGF-B gene. Cancer Cell. 2007;11:147-60.

39. Iwadate $Y$, Matsutani $T$, Hirono S, Shinozaki N, Saeki N. Transforming growth factor- $\beta$ and stem cell markers are highly expressed around necrotic areas in glioblastoma. J Neurooncol. 2016;129:101-7.

40. Guerrero PA, Tchaicha JH, Chen Z, Morales JE, McCarty N, Wang $Q$, et al. Glioblastoma stem cells exploit the $\alpha v \beta 8$ integrinTGF $\beta 1$ signaling axis to drive tumor initiation and progression. Oncogene. 2017;36:6568-80. 
41. Peñuelas S, Anido J, Prieto-Sánchez RM, Folch G, Barba I, Cuartas I, et al. TGF-beta increases glioma-initiating cell selfrenewal through the induction of LIF in human glioblastoma. Cancer Cell. 2009;15:315-27.

42. Hardee ME, Marciscano AE, Medina-Ramirez CM, Zagzag D, Narayana A, Lonning SM, et al. Resistance of glioblastomainitiating cells to radiation mediated by the tumor microenvironment can be abolished by inhibiting transforming growth factor- $\beta$. Cancer Res. 2012;72:4119-29.

43. Bayin NS, Ma L, Thomas C, Baitalmal R, Sure A, Fansiwala K, et al. Patient-specific screening using high-grade glioma explants to determine potential radiosensitization by a TGF- $\beta$ small molecule inhibitor. Neoplasia. 2016;18:795-805. 